\title{
Journal of Real-Time Image Processing: sixth issue of volume 16
}

\author{
Matthias F. Carlsohn ${ }^{1}$ - Nasser Kehtarnavaz ${ }^{2}$ \\ Published online: 12 November 2019 \\ (c) Springer-Verlag GmbH Germany, part of Springer Nature 2019
}

This issue is the last issue of Journal of Real-Time Image Processing in 2019 in which a total of 154 articles were published in order to substantially lower the backlog of Online First articles and to bring forward the Online First articles to February 2018. Starting in 2020, a new publishing structure will be put in place by Springer, which is going to consist of 6 issues per year with the budget of 15 articles per issue.

It is gratifying to see that the reputation and recognition of JRTIP has been steadily growing with its 2018 impact factor standing at 2.588 and its 5 year impact factor standing at 2.378. The journal growth is also reflected in the annual downloads from SpringerLink from 76,000+ downloads in 2017 to $81,000+$ downloads in 2018 . We are grateful to the journal editors for all their contributions processing nearly 450 articles from which about 90 articles were accepted. We are also thankful to the reviewers who provided expert reviews for the submitted articles. Our hope is that the new publishing structure will further improve the operation of the journal.

Various issues related to the operation of the journal were discussed during the JRTIP editorial board meeting in Baltimore in April 2019 during the SPIE Conference on Realtime Image Processing and Deep Learning (see Fig. 1). In particular, it was discussed ways to shorten the time duration of the review process while at the same time lowering the reviewing load for the editors and reviewers. As a solution, it was decided to limit the number of pages for submitted manuscripts to 12 double column pages beginning in Jan 2020 . The journal webpage and manuscript submission instructions have thus been modified to convey this page limit to authors. On behalf of the editorial team, we would like to

Matthias F. Carlsohn

Matthias.Carlsohn@t-online.de

$\bowtie$ Nasser Kehtarnavaz

kehtar@utdallas.edu

1 Engineering and Consultancy for Computer Vision and Image Communication, Bremen, Germany

2 University of Texas at Dallas, Richardson, TX, USA

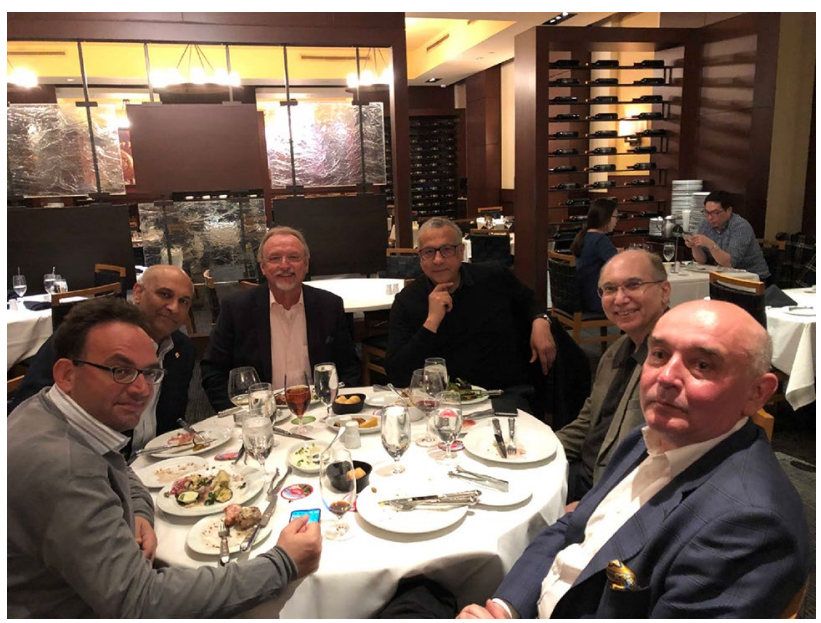

Fig. 1 Meeting of the JRTIP editorial board in April 2019. Members in attendance from left to right: Sergio Saponara, Mukul Shirvaikar, Matthias Carlsohn, Mohamed Akil, Nasser Kehtarnavaz, and Bogdan Smolka

express our gratitude to all the stakeholders for making the journal a key reference in real-time image processing.

Similar to previous years, the editorial board of JRTIP will be meeting to discuss various operational issues related to the journal during the SPIE Conference on Real-Time Image Processing and Deep Learning (whose call for papers appears in the backmatter of this issue) in April 2020 in Anaheim as part of the SPIE Symposium on Defense and Commercial Sensing.

This sixth issue in 2019 comprises 34 original research papers with one being a survey paper entitled "Techniques of medical image processing and analysis accelerated by highperformance computing: a systematic literature review". The papers in this issue cover a variety of applications where robust image processing solutions are developed to enable processing of image data under real-time constraints.

Currently, several calls for papers are active which are included in the backmatter of this issue. 
Finally, we would like to point out that the publisher Springer has put together a new look for the journal website at http://www.springer.com/journal/11554. In this new look, the special issue calls for papers are made more clearly visible which can also be viewed through this link https://www. springer.com/journal/11554/updates. We would like to thank Springer for their efforts in designing the new look for the journal website.
Publisher's Note Springer Nature remains neutral with regard to jurisdictional claims in published maps and institutional affiliations. 